\section{Selektiver COX-2-Inhibitor als Generikum verfügbar}

Seit Juni ist mit Etori 1 A Pharma ${ }^{\circledR}$ eine genersche Alternative zu Arcoxia ${ }^{\circledR}$ verfügbar. Etoricoxib gehört zur Gruppe der Coxibe bzw. der selektiven COX-2-Hemmer. Das Coxib ist indikationsgleich zum Erstanbieter. Coxibe sind eine Weiterentwicklung der herkömmlichen nicht-steroidalen Antirheumatika (NSAR). Sie hemmen nur das Enzym COX-2, das bei Entzündungen eine Rolle spielt, und wirken so entzündungshemmend und schmerzlindernd. Das Enzym COX-1, das für den Schleimhautschutz und die Blutgerinnungsfähigkeit zuständig ist, wird nicht gehemmt. Der Vorteil der Coxibe gegenüber anderen NSAR ist daher, dass sie das Risiko für gastrointestinale Blutungen reduzieren. Zudem beeinflussen sie die Blutgerinnungsfähigkeit nicht. Weitere Informationen zum Produkt sind unter www.1apharma.de verfügbar. - Red.

- Nach Informationen von 1 A Pharma

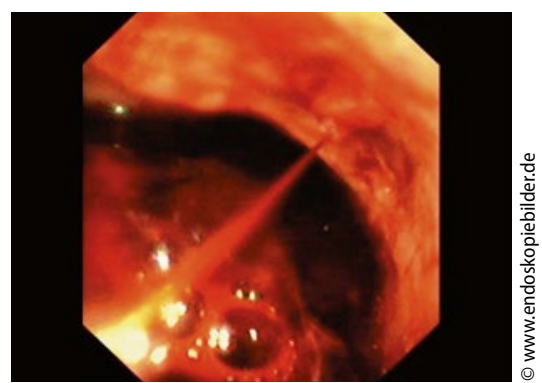

Arterielle Blutung aus einem Magengeschwür - unter einem Cox-2Hemmer ist das deutlich seltener.

\section{Neues Azol gegen systemische Mykosen}

Knochenmarktransplantierte und Keukämie-Patienten haben ein hohes Risiko, an invasiven Pilzinfektionen zu erkranken. Das Azol Isavuconazol (Cresemba ${ }^{\oplus}$ ) hat in der SECURE-Studie gleichwertige Überlebensdaten gebracht wie Voriconazol. Isavuconazol lässt sich auch unabhängig von den Mahlzeiten einnehmen. Es ist gut nierenverträglich und kann selbst bei Dialysepatienten eingesetzt werden. Ob Hauthypersensitivitäten bei dem neuen Azol ein Thma sind, lässt sich serzeit noch nicht abschließend beurteilen.

- Red.

\title{
Glukosesenkung ist nicht alles
}

_Als weiterhin wichtigsten Blutzuckerparameter zur Therapiesteuerung betrachtet Prof. Stephan Jacob, Villingen-Schwenningen, den $\mathrm{HbA}_{1 c^{-}}$ Wert. „Der $\mathrm{HbA}_{1 \mathrm{c}}$-Wert ist nicht ganz perfekt, da er nur die Hyperglykämien der letzten drei Monate anzeigt", sagte Jacob. Außerdem erfasse er keine Hypoglykämien, hyperglykämischen Spitzen und die mittlere Amplitude der Glukoseauslenkungen. Damit eigne sich der $\mathrm{HbA}_{1 \mathrm{c}}$-Wert nicht zur kurzfristigen Therapiesteuerung. Allerdings bleibe er in den Leitlinien ein relevanter Parameter, da er einfach zu messen sei, Informationen zur Glukoselast liefere und eine Einordnung der Gefährdung aus der Epidemiologie erlaube. Ein $\mathrm{HbA}_{1 \mathrm{c}}$-Wert von $6,5-7,5 \%$ als Therapieziel bei Diabetes werde in allen Leitlinien empfohlen, ebenso wie ein LDL-
Cholesterin-Wert von $<70$ mg/dl [Capatano AL et al. Eur Heart J. 2016; 37:29993058].

Dabei habe sich der PCSK9-Hemmer Alirocumab (Praluent ${ }^{\circledR}$ ) als effektiv in der LDL-C-Senkung erwiesen, bemerkte Jacob. Ergebnissen der ODYSSEYDM-INSULIN-Studie zufolge, die bei der diesjährigen Jahrestagung der American Diabetes Association (ADA) präsentiert wurden, hätten $76,4 \%$ der Studienteilnehmer unter Alirocumab (75 mg, evtl. auftitriert auf $150 \mathrm{mg} / \mathrm{Wo}$ che) innerhalb von 24 Wochen einen LDL-C-Wert von $<70 \mathrm{mg} / \mathrm{dl}$ erreicht. In der Placebogruppe war dies nur bei 7,4\% der Teilnehmer der Fall.

Anne Bäurle

- Pressekonferenz „Diabetes to go - Diabetesmanagement: mehr als nur Blutzuckerkontrolle?"; Eppstein-Bremthal, August 2017 (Veranstalter: Sanofi-Aventis)

\section{Funktionelle Magen-Darm-Erkrankungen}

\section{Phytotherapie statt PPI}

_ Zeigen sich Protonenpumpeninhibitoren (PPI) nicht ausreichend wirksam, setzen laut der Umfrage des Kompetenzteams Magen über 70\% der Ärzte Phytopharmaka ein - entweder als Ergänzung oder als Ersatz. Phytotherapeutika mit Multi-Target-Ansatz wie die Iberis-amara-Kombination (Iberogast ${ }^{\circledast}$ ) haben sich vor allem bei funktionellen MagenDarm-Erkrankungen wie der funktionellen Dyspepsie als effektive und nebenwirkungsarme Alternative bewährt.

Die neun Heilpflanzen greifen parallel an unterschiedlichen pharmakologischen Zielen an, z. B. an Kalziumkanälen, afferenten Nervenfasern und cholinergen Neuronen in Muskelzellen. So können die gestörte Motilität normalisiert, die Hypersensibilität verringert, die Säureproduktion gehemmt und Entzündungen entgegengewirkt werden. Der Muskel- tonus im unteren Ösophagussphinkter nimmt zu, in Fundus und Korpus wird eine Relaxation beobachtet, im Antrum kommt es dagegen zu einer Motilitätssteigerung. Die Inhaltsstoffe sind schnell am Wirkort und können die Beschwerden bereits nach einigen Minuten vermindern [Vinson BR, Holtmann G. Digestive Disease Week. 2013; Abstract \#1881].

Iberogast ${ }^{\circledast}$ ist das einzige in Deutschland für die Behandlung der funktionellen Dyspepsie zugelassene Phytotherapeutikum. Es wurde von der Expertenkommission der DGVS (Deutsche Gesellschaft für Gastroenterologie, Verdauungs- und Stoffwechselkrankheiten) in die Leitlinien und Therapieempfehlungen zu funktionellen Dyspepsie aufgenommen.

Red. 